Supplementary Information

\title{
Humidity dependence of the condensational growth of $\alpha$-pinene secondary organic aerosol particles
}

Yiming Qin (1), Jianhuai Ye (1), Paul Ohno (1), Jinghao Zhai (1,2), Yuemei Han (1), Pengfei Liu (1,3), Junfeng Wang (1), Rahul A. Zaveri* (4), and Scot T. Martin* $(1,5)$

(1) School of Engineering and Applied Sciences, Harvard University, Cambridge, Massachusetts, 02138, USA

(2) Department of Environmental Science and Engineering, Fudan University, Shanghai 200433, China

(3) School of Earth and Atmospheric Sciences, Georgia Institute of Technology, Atlanta, Georgia, 30332, USA

(4) Atmospheric Sciences and Global Change Division, Pacific Northwest National Laboratory, Richland, WA, 99352, USA

(5) Department of Earth and Planetary Sciences, Harvard University, Cambridge, Massachusetts, 02138, USA 
Supplemental information. The supplemental information includes a description of the MCMSIMPOL simulation (Text S1), experimental conditions and observations (Table S1), summary of the observations of cyclic growth in the Harvard Environmental Chamber (Table S2), parameters of the MCM-SIMPOL simulation (Table S3), results of the MCM-SIMPOL simulation (Table S4), results of the MCM-SIMPOL simulation represented as a volatility basis set (Table S5), full data set that corresponds to Figure 1 (Figure S1), flow chart of MOSAIC simulation (Figure S2), diagnostic scatter plots of the data sets (Figure S3), and data sets at 20\% and $60 \%$ RH that complement the data sets shown in Figure 5 (Figure S4).

\section{S1: MCM-SIMPOL simulation for initial guess}

For an initial guess of the product volatilities to initialize the model, a chemical simulation (MCM-SIMPOL) was used to represent SOA particle production from VOC oxidation in the Harvard Environmental Chamber. The full description of the simulation approach appears in Chen et al. ${ }^{1}$ In brief, the oxidation products at steady state were obtained by use of the Master Chemical Mechanism (314 species, 942 reactions, selected from MCM, version 3.2) for the chamber conditions of $\alpha$-pinene ozonolysis (Tables S3 and S4). ${ }^{1}$ The saturation concentrations of the produced species were estimated using a group contribution method (SIMPOL). ${ }^{2}$ The MCM-SIMPOL results of the individual products were mapped onto the volatility-basis-set for use in the MOSAIC simulation (Table S5). ${ }^{3}$ The products included semi- and intermediate-volatility compounds as well as low-volatility compounds. The products were distributed across decadal saturation concentrations from $10^{-1}$ to $10^{3} \mu \mathrm{g} \mathrm{m}^{-3}$ (Table S5). These values served as an initial guess for use in MOSAIC. The chemistry in MCM is incomplete and the vapor pressure estimates of SIMPOL are not completely accurate. 
Simulation conditions used herein, together with those of similar studies by Shilling et al. ${ }^{4}$ and Chen et al., ${ }^{1}$ are summarized in Table S3. Production of SOA particle mass concentration was treated by absorptive partitioning governed by a net rate of condensation. ${ }^{5}$ The percent yields of individual products from the MCM-SIMPOL simulation and the partitioning into the gas and particle phases are listed in Table S4. The simulated SOA particle mass concentration was within $10 \%$ of the measured concentration. The simulated and measured $\mathrm{H}: \mathrm{C}$ and O:C ratios agreed within \pm 0.1 (Table $\mathrm{S} 3$ ).

S2: The governing equations for size-dependent gas-particle partitioning in MOSAIC

The rate of change of the average concentration $\bar{C}_{a, i, m}$ ( $\mathrm{mol} \mathrm{cm}^{-3}$ (air)) of solute $i$ in size bin m of radius $R_{p, m}(\mathrm{~cm})$ with number concentration $N_{m} \overline{\left(\mathrm{cm}^{-3}\right)}$ due to mass transfer between gas and particle is given as follows:

$$
\frac{d \bar{C}_{a, i, m}}{d t}=4 \pi R_{p, m}^{2} N_{m} K_{g, i, m}\left(\bar{C}_{g, i}-\frac{\bar{C}_{a, i, m}}{\sum_{j} \bar{C}_{a, j, m}} C_{g, i}^{*}\right)
$$

where $C_{g, i}\left(\mathrm{~mol} \mathrm{~cm}^{-3}\right)$ is the average bulk gas-phase concentration of the solute and $C_{g, i}^{*}$ (mol $\left.\mathrm{cm}^{-3}\right)$ is its effective saturation vapor concentration. $K_{g, i, m}\left(\mathrm{~cm} \mathrm{~s}^{-1}\right)$ is the overall gas-side mass transfer coefficient given by:

$$
\underline{\frac{1}{K_{g, i, m}}}=\frac{1}{k_{g, i, m}}+\frac{1}{k_{p, i, m}}\left(\frac{C_{g, i}^{*}}{\rho_{\mathrm{molar}, m}}\right)
$$

where $k_{g, i, m}\left(\mathrm{~cm} \mathrm{~s}^{-1}\right)$ is the gas-side mass-transfer coefficient given by Eq. (S3), $k_{p, i, m}\left(\mathrm{~cm} \mathrm{~s}^{-1}\right)$ is the particle-side mass transfer coefficient given by Eq. (S4), and $\rho_{\text {molar }, m}$ is the molar density of the organic phase $\left(\mathrm{mol} \mathrm{cm}^{-3}\right.$ (particle)).

$$
\begin{gathered}
k_{g, i, m}=\frac{D_{g, i}}{R_{p, m}} f\left(K n_{i, m}, \alpha_{i}\right) \\
k_{p, i, m}=5 \frac{D_{b, i}}{R_{p, m}}
\end{gathered}
$$


where $f\left(K n_{i, m}, \alpha_{i}\right)$ is the transition regime correction factor to the Maxwellian flux as a function of the Knudsen number $K n_{i, m}=\lambda_{i} / R_{p, m}$ (where $\lambda_{i}$ is the mean free path) and the mass accommodation coefficient, $\alpha_{i}$, which is defined as the fraction $\left(0 \leq \alpha_{i} \leq 1\right)$ of the incoming molecules that is incorporated into the particle surface:

$$
f\left(K n_{i, m}, \alpha_{i}\right)=\frac{0.75 \alpha_{i}\left(1+K n_{i, m}\right)}{K n_{i, m}\left(1+K n_{i, m}\right)+0.283 \alpha_{i} K n_{i, m}+0.75 \alpha_{i}}
$$

\section{References}

1. Chen, Q.; Liu, Y.; Donahue, N. M.; Shilling, J. E.; Martin, S. T., Particle-phase chemistry of secondary organic material: Modeled compared to measured O:C and $\mathrm{H}: \mathrm{C}$ elemental ratios provide constraints. Environ. Sci. Technol., 2011, 45, 4763-4770, DOI: 10.1021/es104398s.

2. Pankow, J. F.; Asher, W. E., Simpol.1: A simple group contribution method for predicting vapor pressures and enthalpies of vaporization of multifunctional organic compounds. Atmos. Chem. Phys., 2008, 8, 2773-2796, DOI: 10.5194/acp-8-2773-2008.

3. Donahue, N. M.; Robinson, A. L.; Stanier, C. O.; Pandis, S. N., Coupled partitioning, dilution, and chemical aging of semivolatile organics. Environ. Sci. Technol., 2006, 40, $2635-$ 2643, DOI: 10.1021/es052297c.

4. Shilling, J. E.; Chen, Q.; King, S. M.; Rosenoern, T.; Kroll, J. H.; Worsnop, D. R.; DeCarlo, P. F.; Aiken, A. C.; Sueper, D.; Jimenez, J. L.; Martin, S. T., Loading-dependent elemental composition of $\alpha$-pinene SOA particles. Atmos. Chem. Phys., 2009, 9, 771-782, DOI: 10.5194/acp-9-771-2009.

5. Seinfeld, J. H.; Pandis, S. N., Atmospheric Chemistry and Physics: From Air Pollution to Climate Change. 3rd ed.; Wiley: Hoboken, NJ, 2016.

6. Han, Y.; Gong, Z.; Liu, P.; de Sá, S. S.; McKinney, K. A.; Martin, S. T., Influence of particle surface area concentration on the production of organic particulate matter in a continuously mixed flow reactor. Environ. Sci. Technol., 2019, 53, 4968-4976, DOI: 10.1021/acs.est.8b07302. 
Table S1. Summary of experimental conditions and observations.

\begin{tabular}{cccc}
\hline $\begin{array}{c}\text { Relative humidity } \\
(\%)\end{array}$ & $\begin{array}{c}\text { Ozone concentration } \\
(\mathrm{ppb})\end{array}$ & $\begin{array}{c}\text { Particle number } \\
\text { concentration } \\
\left(\mathrm{cm}^{-3}\right)\end{array}$ & $\begin{array}{c}\text { Particle area } \\
\text { concentration } \\
\left(\mathrm{nm}^{2} \mathrm{~cm}^{-3}\right)\end{array}$ \\
\hline 0 & $341 \pm 10$ & $3517 \pm 58$ & $(4.9 \pm 0.1) \times 10^{8}$ \\
20 & $338 \pm 7$ & $3813 \pm 83$ & $(5.1 \pm 0.1) \times 10^{8}$ \\
40 & $337 \pm 7$ & $3689 \pm 78$ & $(5.0 \pm 0.1) \times 10^{8}$ \\
60 & $324 \pm 4$ & $3538 \pm 109$ & $(5.1 \pm 0.1) \times 10^{8}$ \\
75 & $302 \pm 9$ & $3465 \pm 135$ & $(5.1 \pm 0.2) \times 10^{8}$ \\
\hline
\end{tabular}


Table S2. Summary of observed occurrence or not of cyclic growth in the Harvard Environmental Chamber. *Unpublished results.

\begin{tabular}{|c|c|c|c|c|c|c|c|c|c|c|c|}
\hline & $\begin{array}{l}\text { Cyclic } \\
\text { growth? }\end{array}$ & $\begin{array}{l}\alpha \text {-pinene } \\
(\mathrm{ppb})\end{array}$ & Oxidant & $\begin{array}{c}\text { Relative } \\
\text { humidity } \\
(\%)\end{array}$ & $\begin{array}{c}\text { Temperatur } \\
\mathrm{e} \\
\left({ }^{\circ} \mathrm{C}\right)\end{array}$ & $\begin{array}{c}\text { Seed } \\
\text { particle }\end{array}$ & $\begin{array}{c}\text { Inflow } \\
\text { number } \\
\text { concentrati } \\
\text { on } \\
\left(\mathrm{cm}^{-3}\right)\end{array}$ & $\begin{array}{c}\text { Inflow } \\
\text { particle } \\
\text { area } \\
\text { concentrati } \\
\text { on } \\
\left(\mathrm{nm}^{2} \mathrm{~cm}^{-3}\right)\end{array}$ & $\begin{array}{l}\text { Chamber } \\
\text { residence } \\
\text { time } \\
\text { (h) }\end{array}$ & $\begin{array}{c}\text { Outflow } \\
\text { number } \\
\text { concentrati } \\
\text { on } \\
\left(\mathrm{cm}^{-3}\right)\end{array}$ & $\begin{array}{c}\text { Outflow } \\
\text { particle } \\
\text { area } \\
\text { concentrat } \\
\text { on } \\
\left(\mathrm{nm}^{2} \mathrm{~cm}^{-3}\right)\end{array}$ \\
\hline This Study & Yes & 22 & $\begin{array}{c}\mathrm{O}_{3} \\
(350 \mathrm{ppb})\end{array}$ & $0-75$ & 22 & None & 0 & 0 & 4.4 & 3564 & $5 \times 10^{8}$ \\
\hline $\begin{array}{c}\text { Vercruysse } \\
\text { et al.* }\end{array}$ & Yes & 20 & $\begin{array}{c}\mathrm{O}_{3} \\
(300 \mathrm{ppb})\end{array}$ & $0-60$ & 22 & None & 0 & 0 & 4.4 & 3500 & $5 \times 10^{8}$ \\
\hline $\begin{array}{c}\text { Aruffo et } \\
\text { al. }^{*}\end{array}$ & Yes & 40 & $\mathrm{OH}$ & $5-80$ & 22 & None & 0 & 0 & 4.9 & 800 & $5 \times 10^{9}$ \\
\hline Han et al. ${ }^{6}$ & Yes & 22 & $\begin{array}{c}\mathrm{O}_{3} \\
(300 \mathrm{ppb})\end{array}$ & 40 & 22 & $\left(\mathrm{NH}_{4}\right)_{2} \mathrm{SO}_{4}$ & $\mathrm{n} / \mathrm{a}$ & $\mathrm{n} / \mathrm{a}$ & 4.5 & $\begin{array}{l}4200- \\
4500\end{array}$ & $\begin{array}{c}(5.5-6.0) \\
\times 10^{8}\end{array}$ \\
\hline Han et al. ${ }^{6}$ & No & 22 & $\begin{array}{c}\mathrm{O}_{3} \\
(300 \mathrm{ppb})\end{array}$ & 40 & 22 & $\left(\mathrm{NH}_{4}\right)_{2} \mathrm{SO}_{4}$ & $\mathrm{n} / \mathrm{a}$ & $\mathrm{n} / \mathrm{a}$ & 4.5 & $\begin{array}{l}8200- \\
49000\end{array}$ & $\begin{array}{c}(0.93- \\
2.87) \times 10^{5}\end{array}$ \\
\hline $\begin{array}{c}\text { Shilling et } \\
\text { al. }^{4}\end{array}$ & No & $0.9-91$ & $\begin{array}{c}\mathrm{O}_{3} \\
(50-535 \\
\text { ppb) }\end{array}$ & 40 & 25 & $\left(\mathrm{NH}_{4}\right)_{2} \mathrm{SO}_{4}$ & $5000-8000$ & $\begin{array}{c}(0.6-1.1) \\
\times 10^{8}\end{array}$ & 3.4 & $\mathrm{n} / \mathrm{a}$ & $\mathrm{n} / \mathrm{a}$ \\
\hline
\end{tabular}


Table S3. Summary of experimental conditions used for the MCM-SIMPOL simulations of SOA production in the Harvard Environmental Chamber.

\begin{tabular}{|c|c|c|c|c|c|c|c|c|c|c|c|c|}
\hline & \multicolumn{6}{|c|}{ Conditions } & \multicolumn{6}{|c|}{ Particle-phase products } \\
\hline & \multirow[b]{2}{*}{$\begin{array}{c}\alpha \text {-pinene } \\
(\mathrm{ppbv})\end{array}$} & \multirow[b]{2}{*}{$\begin{array}{c}\mathrm{O}_{3} \\
(\mathrm{ppbv})\end{array}$} & \multirow[b]{2}{*}{$\begin{array}{l}\text { RH } \\
(\%)\end{array}$} & \multirow[b]{2}{*}{$\begin{array}{l}\text { Temp } \\
\left({ }^{\circ} \mathrm{C}\right)\end{array}$} & \multirow[b]{2}{*}{$\begin{array}{l}\text { Residence } \\
\text { time }(\mathrm{h})\end{array}$} & \multirow{2}{*}{$\begin{array}{c}\text { Seed } \\
\text { and } \\
\text { OH } \\
\text { scavenger }\end{array}$} & \multicolumn{3}{|c|}{ Measured } & \multicolumn{3}{|c|}{ Modeled } \\
\hline & & & & & & & $\begin{array}{c}M_{\text {org }} \\
(\mu \mathrm{g} \\
\left.\mathrm{m}^{-3}\right) \\
\end{array}$ & $\mathrm{H}: \mathrm{C}$ & $\mathrm{O}: \mathrm{C}$ & $\begin{array}{c}M_{\text {org }} \\
(\mu \mathrm{g} \\
\left.\mathrm{m}^{-3}\right) \\
\end{array}$ & $\mathrm{H}: \mathrm{C}$ & $\mathrm{O}: \mathrm{C}$ \\
\hline $\begin{array}{l}\text { This } \\
\text { Study }\end{array}$ & 22 & 350 & $\begin{array}{c}0- \\
75 \%\end{array}$ & 22 & 4.3 & - & $\begin{array}{l}19.8- \\
20.7\end{array}$ & $\begin{array}{l}1.48- \\
1.51\end{array}$ & $\begin{array}{c}0.51- \\
0.54\end{array}$ & 22.6 & 1.6 & 0.5 \\
\hline $\begin{array}{l}\text { Shilling et } \\
\text { al. }{ }^{4}\end{array}$ & $0.9-91$ & 300 & $40 \%$ & 25 & 3.4 & Yes & $\begin{array}{c}0.5- \\
140\end{array}$ & $\begin{array}{c}1.38- \\
1.51\end{array}$ & $\begin{array}{c}0.29- \\
0.45\end{array}$ & $\mathrm{n} / \mathrm{a}$ & $\mathrm{n} / \mathrm{a}$ & $\mathrm{n} / \mathrm{a}$ \\
\hline $\begin{array}{l}\text { Chen et } \\
\text { al. }{ }^{1}\end{array}$ & $4.5-160$ & 300 & 40 & 25 & 3.4 & Yes & $\begin{array}{c}0.5- \\
140\end{array}$ & $\begin{array}{c}1.47- \\
1.56\end{array}$ & $\begin{array}{c}0.33- \\
0.52\end{array}$ & $\begin{array}{c}0.1- \\
314.5\end{array}$ & $\begin{array}{c}1.6- \\
1.7\end{array}$ & $\begin{array}{c}0.4- \\
0.8\end{array}$ \\
\hline
\end{tabular}


Table S4. MCM-SIMPOL simulation of $\alpha$-pinene ozonolysis. Gas-phase and particle-phase molecular products are listed. Percent is on a carbon-count basis. Reaction conditions used in the simulation are listed in Table S3. The approach for gas-particle partitioning of products is described in Chen et al. ${ }^{1}$

\begin{tabular}{|c|c|c|c|c|c|}
\hline \multicolumn{3}{|c|}{ Gas-phase products } & \multicolumn{3}{|c|}{ Particle-phase products } \\
\hline MCM Species & Formula & Percent $(\%)$ & MCM Species & Formula & Percent $(\%)$ \\
\hline PINAL & $\mathrm{C}_{10} \mathrm{H}_{16} \mathrm{O}_{2}$ & 16.8 & PINIC & $\mathrm{C}_{9} \mathrm{H}_{14} \mathrm{O}_{4}$ & 17.2 \\
\hline $\mathrm{C} 107 \mathrm{OH}$ & $\mathrm{C}_{10} \mathrm{H}_{17} \mathrm{O}_{3}$ & 5.6 & $\mathrm{C} 108 \mathrm{OOH}$ & $\mathrm{C}_{10} \mathrm{H}_{16} \mathrm{O}_{5}$ & 15.7 \\
\hline PINONIC & $\mathrm{C}_{10} \mathrm{H}_{16} \mathrm{O}_{3}$ & 4.6 & $\mathrm{C} 921 \mathrm{OOH}$ & $\mathrm{C}_{9} \mathrm{H}_{16} \mathrm{O}_{5}$ & 7.5 \\
\hline $\mathrm{CH} 3 \mathrm{COCH} 3$ & $\mathrm{C}_{3} \mathrm{H}_{6} \mathrm{O}$ & 4.4 & HOPINONIC & $\mathrm{C}_{10} \mathrm{H}_{16} \mathrm{O}_{4}$ & 7.5 \\
\hline $\mathrm{C} 97 \mathrm{OOH}$ & $\mathrm{C}_{9} \mathrm{H}_{17} \mathrm{O}_{4}$ & 4.3 & $\mathrm{C} 98 \mathrm{OOH}$ & $\mathrm{C}_{9} \mathrm{H}_{16} \mathrm{O}_{5}$ & 6.7 \\
\hline APINBOH & $\mathrm{C}_{10} \mathrm{H}_{18} \mathrm{O}_{2}$ & 4.2 & $\mathrm{C} 812 \mathrm{OH}$ & $\mathrm{C}_{8} \mathrm{H}_{14} \mathrm{O}_{4}$ & 6.4 \\
\hline $\mathrm{C} 96 \mathrm{OH}$ & $\mathrm{C}_{9} \mathrm{H}_{16} \mathrm{O}_{2}$ & 4.0 & $\mathrm{C} 97 \mathrm{OOH}$ & $\mathrm{C}_{9} \mathrm{H}_{16} \mathrm{O}_{4}$ & 5.7 \\
\hline $\mathrm{C} 108 \mathrm{OOH}$ & $\mathrm{C}_{10} \mathrm{H}_{16} \mathrm{O}_{5}$ & 3.9 & $\mathrm{C} 813 \mathrm{OOH}$ & $\mathrm{C}_{8} \mathrm{H}_{14} \mathrm{O}_{6}$ & 5.6 \\
\hline NORPINAL & $\mathrm{C}_{9} \mathrm{H}_{14} \mathrm{O}_{2}$ & 3.6 & $\mathrm{C} 108 \mathrm{OH}$ & $\mathrm{C}_{10} \mathrm{H}_{16} \mathrm{O}_{4}$ & 3.8 \\
\hline $\mathrm{CO} 235 \mathrm{C} 6 \mathrm{CHO}$ & $\mathrm{C}_{7} \mathrm{H}_{8} \mathrm{O}_{4}$ & 3.4 & $\mathrm{C} 922 \mathrm{OOH}$ & $\mathrm{C}_{9} \mathrm{H}_{16} \mathrm{O}_{6}$ & 2.7 \\
\hline $\mathrm{C} 89 \mathrm{CO} 2 \mathrm{H}$ & $\mathrm{C}_{9} \mathrm{H}_{14} \mathrm{O}_{3}$ & 3.3 & $\mathrm{C} 98 \mathrm{OH}$ & $\mathrm{C}_{9} \mathrm{H}_{16} \mathrm{O}_{4}$ & 2.1 \\
\hline $\mathrm{C} 108 \mathrm{O} 2$ & $\mathrm{C}_{10} \mathrm{H}_{15} \mathrm{O}_{5}$ & 3.2 & $\mathrm{C} 812 \mathrm{OOH}$ & $\mathrm{C}_{8} \mathrm{H}_{14} \mathrm{O}_{5}$ & 2.1 \\
\hline APINBCO & $\mathrm{C}_{10} \mathrm{H}_{16} \mathrm{O}_{2}$ & 2.8 & $\mathrm{C} 813 \mathrm{OH}$ & $\mathrm{C}_{8} \mathrm{H}_{14} \mathrm{O}_{5}$ & 2.1 \\
\hline $\mathrm{HCHO}$ & $\mathrm{CH}_{2} \mathrm{O}$ & 2.4 & $\mathrm{C} 813 \mathrm{O} 2$ & $\mathrm{C}_{8} \mathrm{H}_{13} \mathrm{O}_{6}$ & 1.6 \\
\hline $\mathrm{C} 97 \mathrm{O} 2$ & $\mathrm{C}_{9} \mathrm{H}_{16} \mathrm{O}_{4}$ & 1.7 & $\mathrm{C} 107 \mathrm{OH}$ & $\mathrm{C}_{10} \mathrm{H}_{16} \mathrm{O}_{3}$ & 1.5 \\
\hline $\mathrm{C} 107 \mathrm{OOH}$ & $\mathrm{C}_{10} \mathrm{H}_{17} \mathrm{O}_{4}$ & 1.7 & PINONIC & $\mathrm{C}_{10} \mathrm{H}_{16} \mathrm{O}_{3}$ & 1.2 \\
\hline $\mathrm{C} 108 \mathrm{OH}$ & $\mathrm{C}_{10} \mathrm{H}_{16} \mathrm{O}_{4}$ & 1.7 & $\mathrm{C} 97 \mathrm{OH}$ & $\mathrm{C}_{9} \mathrm{H}_{16} \mathrm{O}_{3}$ & 1.2 \\
\hline APINAOOH & $\mathrm{C}_{10} \mathrm{H}_{18} \mathrm{O}_{3}$ & 1.6 & APINBOH & $\mathrm{C}_{10} \mathrm{H}_{18} \mathrm{O}_{2}$ & 1.0 \\
\hline $\mathrm{C} 97 \mathrm{OH}$ & $\mathrm{C}_{9} \mathrm{H}_{16} \mathrm{O}_{3}$ & 1.6 & $\mathrm{C} 922 \mathrm{O} 2$ & $\mathrm{C}_{9} \mathrm{H}_{15} \mathrm{O}_{6}$ & 1.0 \\
\hline $\mathrm{C} 811 \mathrm{OH}$ & $\mathrm{C}_{8} \mathrm{H}_{14} \mathrm{O}_{3}$ & 1.0 & $\mathrm{C} 89 \mathrm{CO} 2 \mathrm{H}$ & $\mathrm{C}_{9} \mathrm{H}_{14} \mathrm{O}_{3}$ & 0.8 \\
\hline $\mathrm{C} 721 \mathrm{CHO}$ & $\mathrm{C}_{8} \mathrm{H}_{12} \mathrm{O}_{3}$ & 1.0 & $\mathrm{C} 107 \mathrm{OOH}$ & $\mathrm{C}_{10} \mathrm{H}_{17} \mathrm{O}_{4}$ & 0.8 \\
\hline C109CO & $\mathrm{C}_{10} \mathrm{H}_{14} \mathrm{O}_{3}$ & 1.0 & $\mathrm{C} 106 \mathrm{OOH}$ & $\mathrm{C}_{10} \mathrm{H}_{16} \mathrm{O}_{5}$ & 0.8 \\
\hline Other products & -- & 14.7 & Other products & -- & 4.8 \\
\hline
\end{tabular}


Table S5. Volatility basis set. The products predicted by the MCM-SIMPOL simulation for $\alpha$-pinene ozonolysis (i.e., Table S3) were binned and mapped as a volatility basis set following the approach of Chen et al. ${ }^{1}$

\begin{tabular}{ccccc}
\hline $\begin{array}{c}\text { Saturation concentration } \\
\left(\mu \mathrm{g} \mathrm{m}^{-3}\right)\end{array}$ & $\begin{array}{c}\text { Average } \\
\text { Molecular } \\
\text { weight } \\
\left(\mathrm{g} \mathrm{mol}^{-1}\right)\end{array}$ & $\begin{array}{c}\text { Gas-phase } \\
\text { mass } \\
\text { concentration } \\
\left(\mu \mathrm{g} \mathrm{m}^{-3}\right)\end{array}$ & $\begin{array}{c}\text { Particle-phase } \\
\text { mass } \\
\text { concentration } \\
\left(\mu \mathrm{g} \mathrm{m}^{-3}\right)\end{array}$ & $\begin{array}{c}\text { Sum concentration } \\
\left(\mu \mathrm{g} \mathrm{m}^{-3}\right)\end{array}$ \\
\hline 0.1 & 203.8 & 0.2 & 2.7 & \\
1 & 186.2 & 0.4 & 4.0 & 4.3 \\
10 & 208.3 & 3.9 & 8.3 & 12.2 \\
100 & 182.8 & 21.5 & 6.3 & 27.8 \\
1000 & 158.0 & 38.1 & 1.4 & 39.5 \\
\hline
\end{tabular}




\section{List of Supplementary Figures}

Figure S1. Full data set that corresponds to Figure 1. See caption there. Figure S2 further shows the stabilization period during the first five days prior to the experiment. The top panel of Figure S1 shows the total surface area concentration as a function of time as well as the time course of the RH profile.

Figure S2. Flow chart of MOSAIC simulation. The initial guess for the volatility of the product distributions was obtained by an MCM-SIMPOL simulation (Section S1).

Figure S3. Diagnostic scatter plots of the data sets. (a) Total number concentration plotted against the mode diameter $\hat{d}_{p}$ of the number-diameter distribution for the data of Figure 1 at each RH. (b) Total area concentration plotted against the mode diameter of the number-diameter distribution for the data of Figure 1 at each RH.

Figure S4. Data sets at $20 \%$ and $60 \%$ RH that complement the data sets shown in Figure 5 . See caption there. 


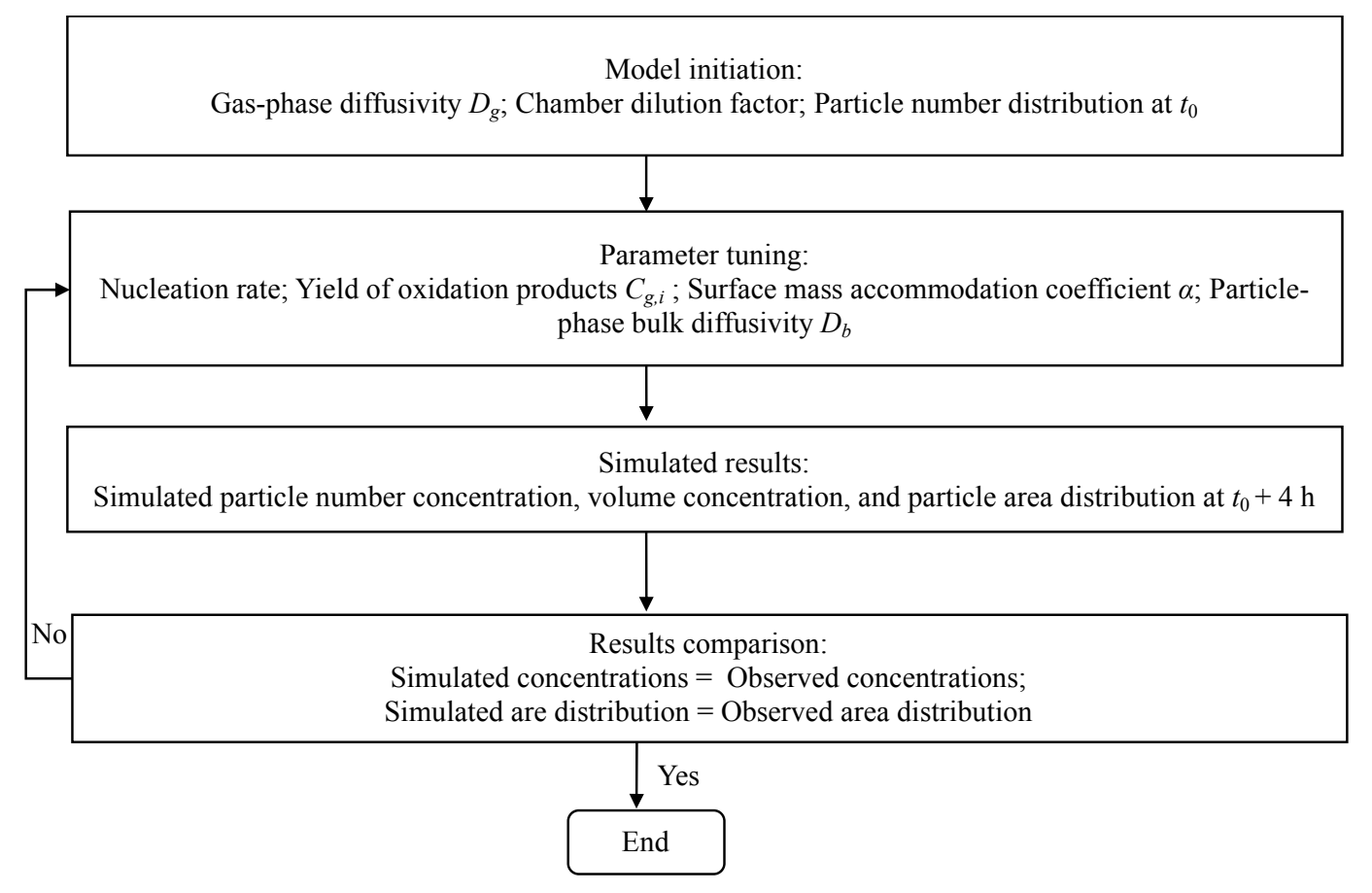

Figure S1 


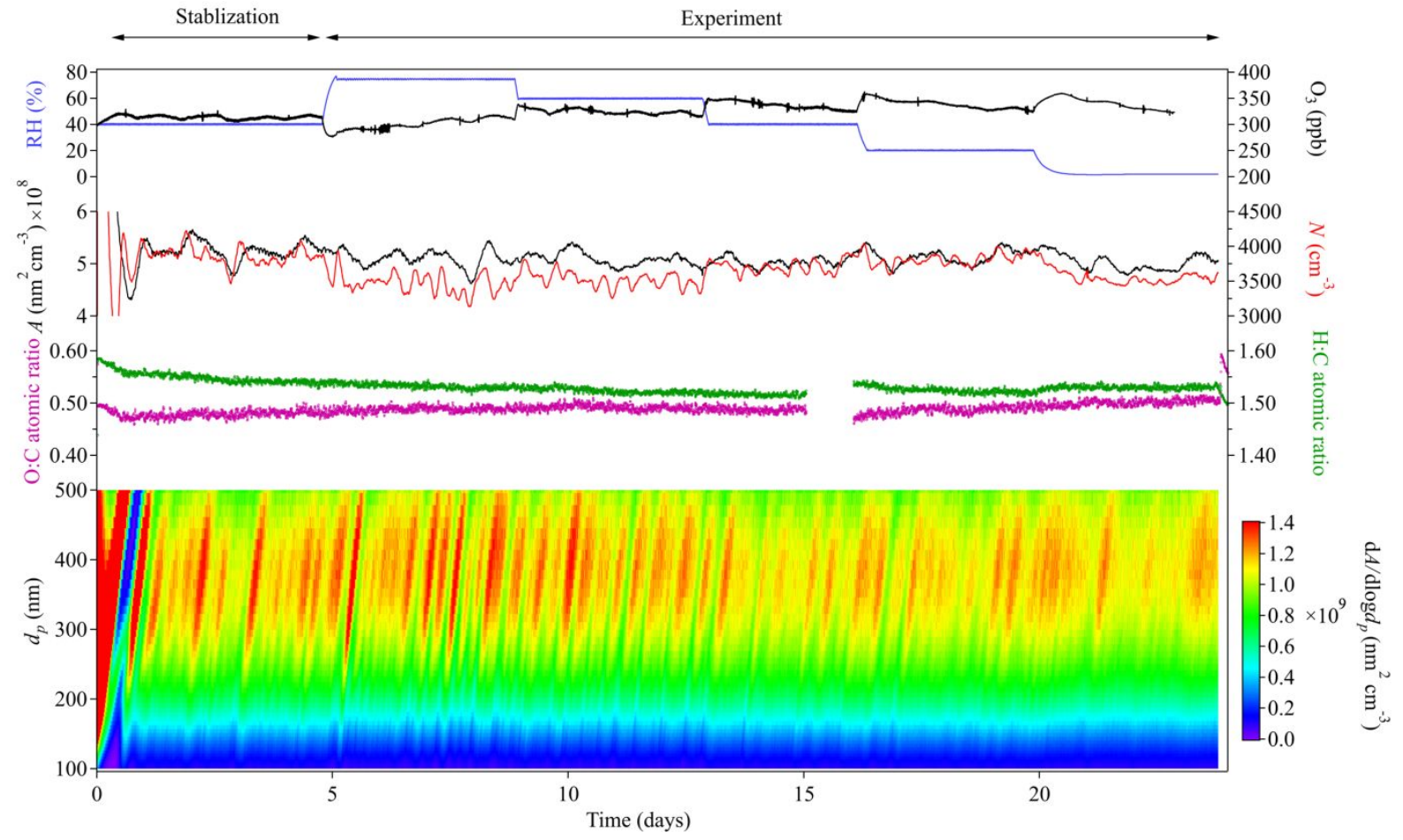

Figure S2 


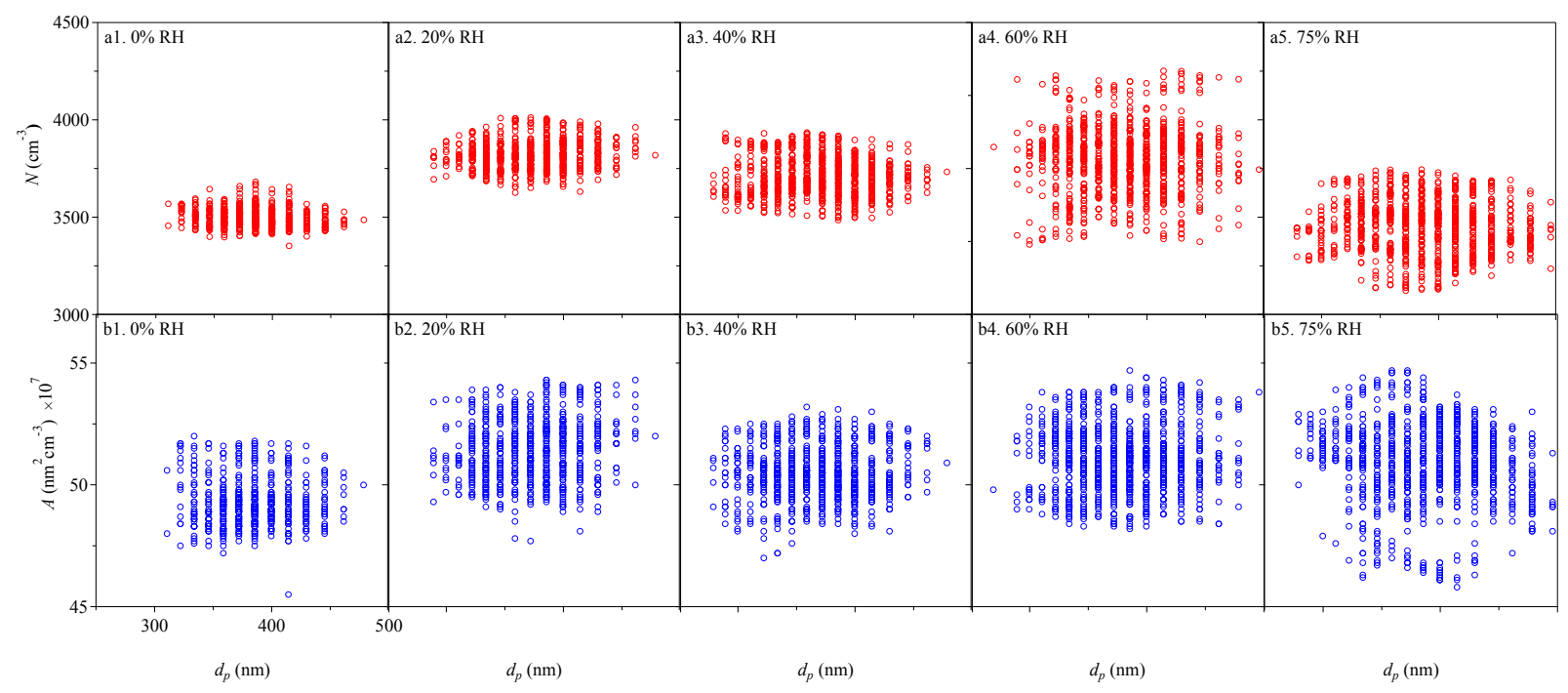

Figure S3 

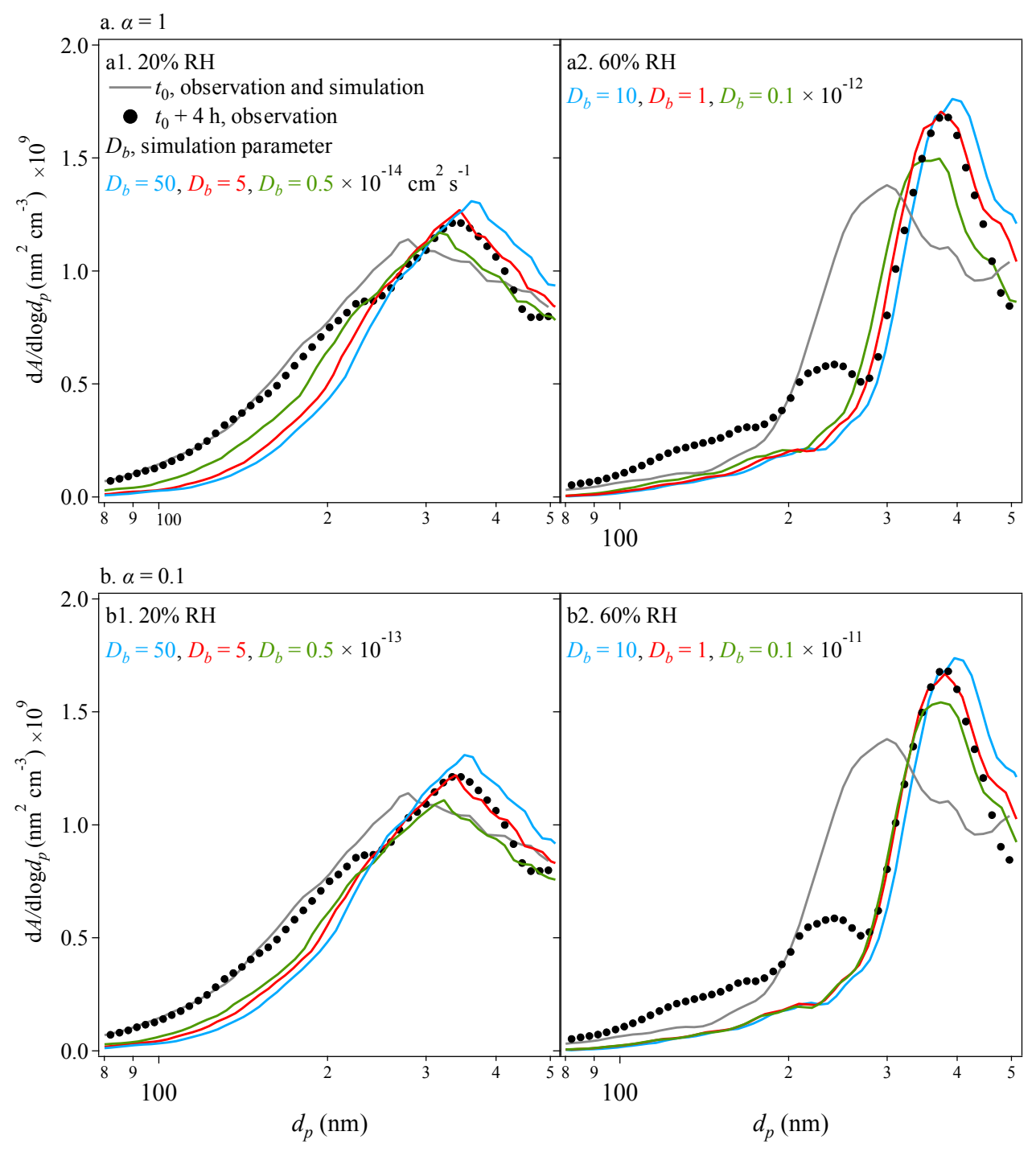

Figure S4 